\title{
ONE-PHONON COHERENT NEUTRON SCATTERING FROM CERTAIN POLYCRYSTALLINE MATERIALS *
}

\author{
W. MYERS, G. C. SUMMERFIELD and J. S. KING \\ Department of Nuclear Engineering, \\ The University of Michigan, Ann Arbor, Michigan
}

Received 22 April 1965

A well-known and useful technique in the study of crystals is the measurement of one-phonon coherent peaks in the scattered neutron spectra from single crystals [1] **. For this case, the cross section is proportional to $\delta\left(\epsilon_{j}(x)-\epsilon\right)$, where $\hbar x$ is the momentum transfer to the neutron, $\epsilon$ is the neutron energy loss and $\epsilon_{j}(x)$ is energy of a phonon with wave vector equal to $x$ in the $j$ 'th branch. If the target is a polycrystalline material, these peaks are generally smeared out and are indistinguishable from the spectrum due to incoherent scattering.

However, as we shall show, there are certain materials, principally high polymers, for which one-phonon, coherent peaks will appear in the neutron spectrum from polycrystalline materials. The one-phonon, coherent neutron cross section from a polycrystalline material is:

$\frac{d^{2} \sigma}{d \Omega d \epsilon}=$

$$
\begin{aligned}
=\frac{k^{\prime}}{k} \sum_{j} \sum_{\tau} \int \mathrm{d}^{3} q \int \mathrm{d} \Omega_{\tau} F(x, q) \rho_{j}(q) \delta\left(\epsilon_{j}(q)-\epsilon\right) \times \\
\times \delta(x+q-2 \pi \tau)
\end{aligned}
$$

where

$$
\begin{aligned}
F(x, q) & =\sum_{s s^{\prime}} a_{s^{\prime}}^{*} a_{s} \exp \left(-2 W_{s s^{\prime}}\right)\left(x \cdot e_{j}^{s}\right)\left(x \cdot e_{j}^{s^{\prime}}\right) \times \\
& \times \frac{(2 \pi)^{3} \hbar^{2} \exp \left\{\mathrm{i} x \cdot\left(r_{s^{\prime}},-r_{s}\right)\right\}}{2 \epsilon_{j}(q) \sqrt{m_{s^{m} m_{s}}}\left|d_{1} \cdot d_{2} \times d_{3}\right|} \frac{1}{1-\exp \left\{-\beta \epsilon_{j}(q)\right\}}
\end{aligned}
$$

and $a_{s}$ is the coherent scattering length for the $s$ 'th atom within the unit cell, $r_{s}$ is the vector

\footnotetext{
* Work supported in part by the United States Atomic Energy Commission and in part by the National Science Foundation.

** We will restrict our attention to downscattering $(\epsilon>0)$ experiments. The upscattering $(\epsilon<0)$ case is essentially the same.
}

locating the $s^{\prime}$ th atom within the unit cell, $d_{1}$, $d_{2}$ and $d_{3}$ are the bravais lattice vectors, $e_{j} s$ is the phonon polarization vector, $\exp \left(-2 W_{s s^{\prime}}\right)$ is the Debye-Waller factor, the $\tau$ are the reciprocal lattice vectors, $\hbar k$ and $\hbar \boldsymbol{k}^{\prime}$ are the initial and final neutron momenta, and $\rho_{j}(q) \mathrm{d}^{3} q$ is the number of phonons with wave vectors in $d^{3} q$ at $q$ in the $j$ 'th mode.

Examining eq. (1), we can see that usually $\mathrm{d}^{2} \sigma / d \Omega d \epsilon$ is a smoothly varying function of $x$ and $\epsilon$.

However, some materials are highly anisotropic, such that the phonon density $\rho_{j}(q)$ can be represented by

$$
\rho_{j}(q)=f_{j}(q) \delta\left(\Omega_{q}-\Omega_{\mathrm{o}}\right)
$$

where $\Omega_{0}$ is a particular direction in a single crystal of the material.

Before exploring the consequences of eq. (2), we should discuss its applicability. In particular, we will consider highly crystalline polymeric chains. For such materials, eq. (2) should hold particularly well. In fact, it has been shown [2] * that, for the optical modes, the normal vibrations of these molecules can be computed assuming the individual molecules are isolated (for which eq. (2) holds exactly). It is possible that eq. (2) can be shown to hold for other types of materials, but none are known to us.

Using eq. (2) in eq. (1), we have +

$$
\frac{\mathrm{d}^{2} \sigma}{\mathrm{d} \Omega \mathrm{d} \epsilon}=\frac{k^{\prime}}{k} \sum_{j} \sum_{\tau} \frac{F(x, q)}{J} f_{j}\left(q_{0}\right) \delta\left(\epsilon_{j}\left(q_{\mathrm{O}}\right)-\epsilon\right),
$$
+ It should be noted here that for eq. (1) to hold the crystal must be large in three dimensions. Thus, intermolecular forces must be present. Such forces are usually present, for example the polyethylene crystal is three dimensional [3]. However, the inter- molecular forces are generally so much weaker than the intramolecular forces that they can be ne- glected for the high energy modes.
$\uparrow+$ Note that $F\left(x, q \Omega_{0}\right)$ is a function only of $x$ and $q$.


where

$$
\left|q_{\mathrm{O}} \Omega_{\mathrm{O}}-2 \pi \tau\right|=\kappa
$$

and $J$ is the Jacobian.

$$
J=\left|\frac{q^{2} \mathrm{~d} q \mathrm{~d} \Omega_{\tau}}{\mathrm{d}^{3}\left(q_{\mathrm{O}} \Omega_{\mathrm{O}}-2 \pi \tau\right)}\right|
$$

Eq. (3) indeed predicts one-phonon peaks in the scattered neutron spectrum of high polymers. However, these materials usually have a large number of hydrogen atoms, and these peaks will be rather difficult to observe due to the large incoherent background. (E.g., the hydrogen cross section is only about $5 \%$ coherent.) Thus, to observe these peaks, the experimental statistics must be extremely good. The fact that the locations of the peaks as a function of $\epsilon$ should vary continuously as a function of $\kappa$, and conversely, should help greatly in verifying their existence.

\section{References}

1. B.N.Brockhouse, Proc. I.A.E.A. Symp. on Inelastic scattering of neutrons, Vienna, 1961.

2. Tasumi, Shimanouchi and Miyazawa, J.Mol. Spect. 9 (1962) 261; 11 (1963) 422;

Miyazawa and Kitagawa, Polymer Letters 2 (1964) 395 ;

Lin and Koenig, J. Mol. Spect. 9 (1962) 228.

3. See e.g., Volkenstein, Configurational statistics of polymeric chains, High Polymers, Vol. XVIII (Interscience Publishers, New York, 1963).

\section{PARAMAGNETIC RELAXATION OF Cr-DOPED SPINEL \\ D. E. DUGDALE *}

The General Electric Company Limited, Central Research Laboratories Hirst Research Centre, Wembley, England

Received 4 May 1965

The spin lattice relaxation of many iron group materials has been found to depend on the paramagnetic concentration (e.g. Gill [1]). Van Vleck's [2] suggested explanation of these effects in terms of cross relaxation to fast relaxing centres, such as ion pairs, has been shown by Elliot and Gill [3] to lead to an inverse square concentration dependence when cross relaxation is the bottleneck process rather than the relaxation of the ion pairs. Under these conditions temperature-independent relaxation may also be observed when only low-lying energy levels of the "pair system" are involved. Relaxation phenomena consistent with this theory have been observed in the $\mathrm{Cr}^{3+}$ spinel system $\left(\mathrm{MgAl}_{2} \mathrm{O}_{4}\right)$.

Using the pulsed saturation technique at 9.2 $\mathrm{Gc} / \mathrm{s}$, the relaxation of the $\left(+\frac{1}{2} \rightarrow-\frac{1}{2}\right)$ transition was investigated with the magnetic field along a trigonal [111] axis. Due to the large zero field splitting parameter $2 \mathrm{D}$ of $+55.5 \mathrm{Gc} / \mathrm{s}$ (Atsarkin [4]) this is the only observable transition.

According to the single ion theory of Mattuck and Strandberg [5] and the results of Emel'yanova

* Now at Department of Applied Physics, University of Durham.

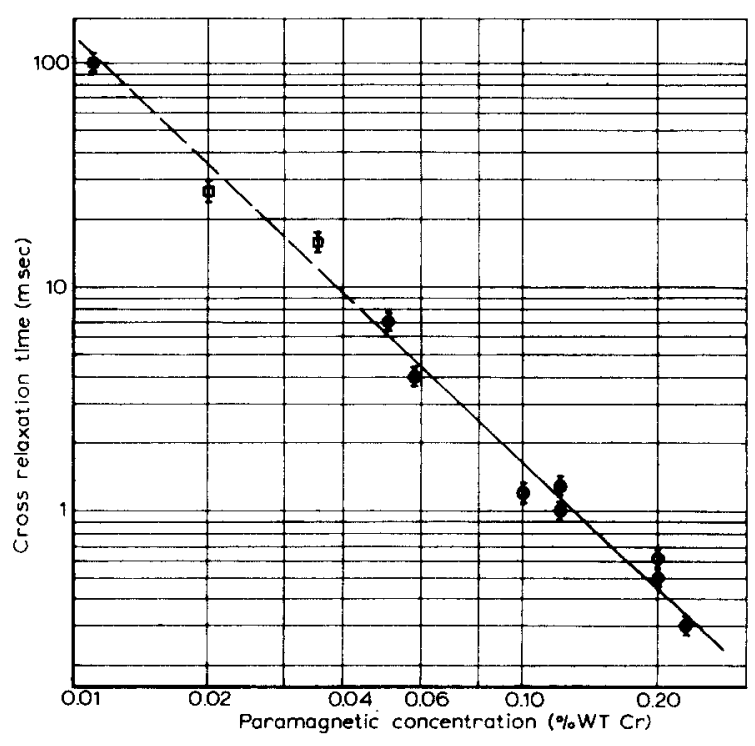

Fig. 1. Plot of cross relaxation time against chromium concentration. $\Phi$ directly observed relaxation times. 中 relaxation times deduced from fig. 2 . 